University of San Diego

Digital USD

Spring 5-27-2017

\title{
Evaluation of Hypertensive Clinical Significance in a Primary Care Setting with Veterans with Diabetes
}

Claire S. Moga MSN, RN

University of San Diego, cmoga-09@sandiego.edu

Follow this and additional works at: https://digital.sandiego.edu/dnp

Part of the Nursing Commons

\section{Digital USD Citation}

Moga, Claire S. MSN, RN, "Evaluation of Hypertensive Clinical Significance in a Primary Care Setting with Veterans with Diabetes" (2017). Doctor of Nursing Practice Final Manuscripts. 55.

https://digital.sandiego.edu/dnp/55

This Doctor of Nursing Practice Final Manuscript is brought to you for free and open access by the Theses and Dissertations at Digital USD. It has been accepted for inclusion in Doctor of Nursing Practice Final Manuscripts by an authorized administrator of Digital USD. For more information, please contact digital@sandiego.edu. 
Evaluation of Hypertensive Clinical Significance in a Primary Care Setting with Veterans with Diabetes

\section{Claire S. Moga, MSN, RN}

Joseph Burkard, DNSc, CRNA

Jian Hua Liu, PhDc, RN

David Bittleman, MD

Hahn School of Nursing and Health Sciences

Beyster Institute for Nursing Research

University of San Diego 


\begin{abstract}
Analyses of hypertensive confounding factors were identified by looking retrospectively at patient demographics, medications, recent blood pressure and the medical history of hypertensive patients with diabetes. The data trends found in this database highlighted potential areas for improvement with regards to the care of patients with this co-morbidity in the outpatient setting. All prescription anti-hypertensives prescribed for the 102 patients followed the National Joint Committee (JNC-8) Hypertension Guideline Algorithm. Of 102 adult males (over the age of 18), 22 patients had a blood pressure that is out of control (over 140/90). Within this group of patients, $33.3 \%$ of the patients are overweight with a body mass index between 25.0 and 29.9. Patients were obese $60.8 \%$ of the time with a BMI of 30.0 and above. Half of the patients (51 of 102) have an active diagnosis of obstructive sleep apnea. There is a need for continued aggressive treatment of hypertension in order to keep blood pressure below the recommended 140/90mmHg per the JNC-8 Hypertension Guidelines for hypertensive and diabetic patients. Several evidence-based practice recommendations were provided to address the clinically significant findings mentioned above.

Keywords: Veteran, essential hypertension, diabetes mellitus, obesity
\end{abstract}


Evaluation of Hypertensive Clinical Significance in a Primary Care Setting with Veterans with Diabetes

\section{Background \& Significance}

According to the Centers for Disease Control, National Vital Statistical Report, there were 30,221 deaths from essential hypertension and hypertensive renal disease in the United States in 2014. Nearly 75 million American adults have high blood pressure, which is approximately one in every three adults. Of the 75 million adults with hypertension, only about $54 \%$ have their hypertension in control. Early treatment of hypertension is especially important in diabetic patients both to prevent cardiovascular disease and to minimize the progression of renal disease and diabetic retinopathy (Gaede, 1999). There are many negative health outcomes associated with elevated blood pressure including the risk for heart disease and stroke.

An estimated $\$ 48.6$ billion dollars is spent yearly on hypertensive disease, which includes the cost of health care services, medications to treat high blood pressure, and missed days of work (Centers for Disease Control). Thus, aggressive treatment of hypertension is not only the best thing for the wellbeing of patients, but it also has an economic burden on our healthcare system and country.

Patients that are overweight or obese are at an increased risk of having hypertensive disease. There is an overwhelming prevalence of both overweight and obese patients in the United States. More than two-thirds of adults in the U.S are overweight or obese according to the Centers for Disease Control. It is known that type-2 diabetes mellitus and hypertension are associated with obesity. Thus, it is important to evaluate 
the blood pressure and confounding factors that are contributing to both hypertension and diabetic discourse.

The Joint National Committee (JNC) published the "2014 Evidence Based Guideline for the Management of High Blood Pressure in Adults", which includes the JNC - 8 Hypertension Guideline Algorithm for treatment of hypertension in various subgroups. The guideline includes recommended lifestyle changes and pharmacotherapy that is based on various co-morbid conditions. This guideline was used as a map for this evidence based project. The current practices of the clinic were evaluated using the guideline as an evidence based tool for best practice.

\section{Evidence Based Practice Model}

The beginning steps of the IOWA model were used to guide the planning of the project. The IOWA model is presented in a flow chat format with many feedback loops to ensure proper direction within a project. This helps to ensure that the user is meeting certain criteria prior to moving too far into the project in order to help guarantee success later on. The starting point of the IOWA model begins with a question concerning clinical practice, either a problem focused trigger or a knowledge-focused trigger. With regards to the evaluation of the hypertensive data set, the project trigger is a knowledgefocused trigger. There is not a direct "problem" that is causing the need for change, but rather, the knowledge that best practice may not be currently adhered to, and we could do better.

The IOWA model also has a question in the beginning of the model that asks whether or not the project is an organizational priority. If the topic or problem is not of importance to the institution, then the model recommends rethinking the project idea 
entirely. This topic was proposed by the overseeing physician and was expressed as a priority. The IOWA model was fitting for planning this particular evidence based practice project and will continue to serve as a beneficial guide for the organization moving forward.

\section{Project Plan Process}

A Nurse Practitioner, Doctor of Philosophy student, accessed an electronic health record at an outpatient Veterans Administration (VA) internal medicine clinic. From the health record, a de-identified clinical database was assessed with hypertensive patient demographics, medical history, family history and co-morbid diagnoses. The demographics included in the database consisted of the following: age, sex, race, religion, and military branch. Social behaviors such as tobacco use and alcohol use were also included. Psychiatric medical history was included only if it was one of the following conditions: major depressive disorder, generalized anxiety disorder, post traumatic stress disorder. The following medical conditions were included, obstructive sleep apnea (OSA), congestive heart failure (CHF), coronary artery disease (CAD), dyslipidemia, chronic kidney disease (CKD), diabetes mellitus (DM), and a history of a transient ischemic attack (TIA) or a cerebrovascular accident (CVA).

The database also included most recent lab values for hemoglobin A1C, HDL, and body mass index (BMI). Antihypertensive medications including dosing prescribed to the patient were included. There was a range from zero to four antihypertensive medications prescribed. Baseline and most recent blood pressure readings were incorporated and used to assess whether or not blood pressure was in control or not. Finally, the database had family history within specific diagnoses that are most related to 
cardiac disease and cardiac risk factors. The following family history was included: hypertension (HTN), cerebrovascular accident (CVA), myocardial infarction (MI), coronary artery disease (CAD), and diabetes mellitus (DM). All of the patients in the database had an ICD-10 billing code of hypertension. By creating data trends and looking at the JNC-8 Guidelines, only certain factors were used in the analysis of confounding factors to hypertension and diabetes.

There were 325 adult patients (over the age of 18) with a diagnosis of hypertension within the provided database during the dates of August $1^{\text {st }}, 2014$ through July $31^{\text {st }}, 2016$. Of the 325 patients, there were 108 patients with an active diagnosis (ICD-10 code) of diabetes mellitus type II. The JNC-8 Hypertension Guideline Algorithm begins by specifying that the tool is written for adults over the age of 18. It begins with the recommendation that lifestyle modifications are implemented, a set blood pressure goal is created and blood pressure lowering medications are started based on the algorithm. The lifestyle changes are comprised of the following: smoking cessation, blood glucose and lipid control, dietary changes and physical activity. Dietary changes including healthy eating in which the Dietary Approaches to Stop Hypertension (DASH) diet is recommended. Moderate alcohol consumption and a reduced sodium intake to no more than $2,400 \mathrm{mg} / \mathrm{day}$. The physical activity recommendations include moderate to vigorous activity 3-4 days a week averaging 40 minutes per session.

Next, the JNC-8 Hypertension Guideline Algorithm differentiates between the general population (no diabetes or CKD) and patients with diabetes or chronic kidney disease (CKD). For the purposes of this project, the patients with the co-morbidity of diabetes were selected. It is critical to prevent chronic kidney disease or renal failure in 
this population. Patients with both hypertensive disease and diabetes are at a significant risk of poor health outcomes.

Male patients were included in the analysis. There were a total of 102 adult male, veteran patients with an active diagnosis of both hypertension and diabetes type II. Within the diabetic/hypertensive co-morbid diagnosis on the algorithm, the blood pressure goal is goal less than 140/90. Of note, patients age 60 and above without diabetes or chronic kidney disease have a more liberal blood pressure goal of less than 150/90. This does not apply for geriatric patients over 60 with diabetes due to the increased cardiovascular risk with this comorbidity. Thus, all patients at any age with both hypertension and diabetes have a blood pressure goal of 140/90 or below.

According to the guideline, patients are then divided into either black or nonblack. This category will help to determine the pharmacotherapy recommendations for the patient. From this point, each of the patients within this co-morbid group, were divided into patients that were either black or non-black per the guideline. There were 83 nonblack patients and nineteen black patients with hypertension and diabetes. The most recent blood pressure was then analyzed to see if the blood pressure is in control $(<140 / 90)$ or out of control $(>140 / 90)$. Of the 83 non-black patients, twenty-one of the patients had a blood pressure that is considered out of range and of the black patients, there was one patient with a blood pressure out of control. The pharmacotherapy that was prescribed to each of the patients with a blood pressure that is out of range was analyzed to ensure that it is following the JNC-8 Hypertension Guideline Algorithm. 


\section{Evaluation \& Results}

\section{Outcomes}

This next section discusses the evidence based practice project outcomes. Blood pressure control, the antihypertensives prescribed to the patient, body mass index, obstructive sleep apnea, and other contributing factors are discussed herein.

Blood Pressure Control. Out of 102 adult males with a diagnosis of diabetes mellitus type 2 and hypertension, twenty-two patients had a blood pressure that is out of control (over 140/90). All prescription antihypertensives followed the National Joint Committee (JNC) Hypertension Guideline Algorithm.

Anti-Hypertensive's Prescribed. All prescription antihypertensives followed the National Joint Committee (JNC) Hypertension Guideline Algorithm. The medications are specific to patients with both hypertension and diabetes. Also, the medication recommendations differ between black and non-black patients. Of the 102 adult males, twenty-two patients had a blood pressure of 140/90 or above. One of the twenty-two patients is a black male, and the other twenty-one patients are considered non-black.

The one black patient with an elevated blood pressure is on two anti-hypertensive medications. The medications prescribed were hydroclorothiazide (HCTZ) $25 \mathrm{mg}$ per day and verapamil 240mg daily. Both medications are according to the JNC guidelines. The twenty-one non-black patients with elevated blood pressure were on varying medication regimens but all pharmacologic therapy was prescribed according to recommendations. Two of the patients were on no medications. Eight of the patients were on single therapy. Four of the patients are on double therapy. Six of the patients are on triple therapy. And, finally, one patient was on quadruple therapy. 
Out of the eight patients on single therapy, six of the patients were prescribed lisinopril, which is an angiotensin-converting-enzyme inhibitor (ace-inhibitor). The dosing ranged from $5 \mathrm{mg}$ to $40 \mathrm{mg}$ per day. One patient was prescribed metoprolol 50mg per day, which is a beta-blocker. The last patient was prescribed a calcium channel blocker (CCB), amlodipine 10mg daily. The recommended therapy in diabetic hypertensive patients is an ace-inhibitor or an angiotensin II receptor blocker (ARB), calcium channel blocker (CCB) or diuretic.

There were four of the twenty-one patients prescribed dual therapy. Patient one was on atenolol $50 \mathrm{mg}$ per day and losartan 100mg per day. Patient two was on metoprolol 100mg per day and lisinopril 10mg per day. Patient three was on HCTZ 25mg daily and losartan 100mg daily. The forth patient was on HCTZ 25mg per day and lisinopril 40mg per day.

Five of the twenty-one patients were prescribed triple therapy. Patient one and two were on the same three medications, although doses varied. The two patients were both on amlodipine (10mg daily), atenolol (25mg and 100mg daily), and lisinopril (40mg every day). The other two patients were prescribed different medication combinations. Patient three was prescribed amlodipine 4mg daily, atenolol 100mg daily and losartan 100mg per day. Patient four was prescribed spironolactone $25 \mathrm{mg}$, furosemide $80 \mathrm{mg}$ and metoprolol 50mg per day. Finally, patient five was on HCTZ 25mg/triamterene $37.5 \mathrm{mg}$, atenolol 50mg daily and lisinopril 40mg a day.

Finally, two of the twenty-one patients were prescribed quadruple therapy. Patient one was prescribed an ace-inhibitor, beta-blocker, diuretic and centrally-acting agent. The specific medications that patient one was prescribed include the following: lisinopril 
40mg per day, atenolol 100mg daily, HCTZ 25mg per day, and clonidine $0.1 \mathrm{mg}$ daily. This combination of medications follows the JNC-8 Guidelines. Patient two was prescribed HCTZ 20mg/lisinopril 20mg, furosemide 20mg daily, and metoprolol 100mg per day. See table 1.

Body Mass Index. Of the 102 patients, $33.3 \%$ of the patients are overweight with a body mass index between 25.0 and 29.9 . And, $60.8 \%$ of these patients were obese with a BMI of 30.0 and above. Therefore, approximately $94 \%$ of these patients are suffering from being either overweight or obese. Only $5.9 \%$ of the patients were considered to have a healthy weight. See figure 1 . With regards to the twenty-two patients with a blood pressure above 140/90, only two out of the twenty-two (9.1\%) patients are at a healthy weight (BMI 18.5 to 24.9). Therefore, eight out of the twenty-two (36.4\%) patients are overweight (BMI 25 to 29.9). Finally, twelve out of the twenty-two (54.5\%) patients are obese (BMI 30 and above). Together, $91 \%$ of the patients with elevated blood pressure are either overweight or obese.

Obstructive Sleep Apnea. Half (50\%) of the patients (51 of out 102) have an active diagnosis of obstructive sleep apnea (OSA). See figure 2. This data is based of an active ICD-10 code within the patient's medical record. The group of patients with elevated blood pressure, over 140/90, 59\% (13/22) had an active diagnosis of obstructive sleep apnea. Therefore, $41 \%(9 / 22)$ of the patients with an elevated blood pressure do not have an active diagnosis of obstructive sleep apnea. These patients are not currently being screened for OSA and therefore, there is an opportunity to screen this patient population. If these patients have OSA, but are not being treated for the disease, it may be contributing to their high blood pressure and even making it more difficult to decrease. A 
prospective cohort study of 1889 participants was conducted by Marin, et al. (2013) and found that "compared with participants without OSA, the presence of OSA was associated with increased adjusted risk of incident hypertension; however, treatment with CPAP therapy was associated with a lower risk of hypertension." In order to compare the prevalence of obstructive sleep apnea in patients with both hypertension and diabetes, an analysis of the number of patients with OSA with hypertension without diabetes was performed. There were 180 patients within the cohort with an active diagnosis of hypertension without diabetes. Of the 180 patients, $75(41.7 \%)$ patients have an active diagnosis of OSA. Consequently, there was a lower incidence of OSA in patients with hypertension and no diabetes.

Other Contributing Factors. After analyzing various other risk factors that could be contributing to the hypertensive and diabetic patient's cardiovascular health, there were a significant number of patients with an active diagnosis of dyslipidemia. Out of the 102 patients, $85.2 \%$ (87) have an active diagnosis of dyslipidemia. Of those patients, seventeen of them have blood pressure that is out of range (17/22 patients) or $77.3 \%$ of the out of control patients also have dyslipidemia, contributing to their overall cardiovascular risk. $16.7 \%(17 / 102)$ have an active diagnosis of coronary artery disease (CAD), and two of the seventeen patients have blood pressure out of range. With regards to congestive heart failure $(\mathrm{CHF}), 4.9 \%(5 / 102)$ of the patients have an active diagnosis of $\mathrm{CHF}$, and all (100\%) are in hypertensive control.

\section{Evidence Based Practice Suggestions}

It is imperative to aggressively treat hypertensive patients to obtain hypertensive control, especially in the setting of type- 2 diabetes mellitus. According to the JNC-8 
Guidelines, patients with an elevated blood pressure, initial presentation and subsequent readings, lifestyle modifications should be reinforced to the patient. "For all persons with hypertension, the potential benefits of a healthy diet, weight control, and regular exercise cannot be overemphasized" (James, et. al, 2014). There are an overwhelming number of patients that are either overweight of obese with the co-morbidity of hypertension and type-2 diabetes mellitus in this group of veteran patients. According to the JNC-8 Guidelines, weight reduction is a priority for hypertensive patients. Specifically, the guideline suggests the DASH diet for hypertensive/diabetic patients. The DASH diet is endorsed by The National Heart, Lung and Blood Institute, the American heart Association (AHA) and the Mayo Clinic. The DASH diet has been proven to decrease systolic and diastolic blood pressure. This is largely due to the diets sodium restriction. Therefore, all of the patients with these ICD-10 codes will be referred for nutritional counseling to obtain education on the DASH diet. The providers at this outpatient clinic were educated on the DASH diet and low-sodium diet (may be done through dietician). Weight management/ reduction in body mass index will be discussed with the patients in this clinic, especially for patients with an elevated blood pressure. Brown et. al (1989) completed a meta-analysis of 89 studies that assessed educational interventions and outcomes specific to weight loss in diabetes care. The studies included demonstrated support for diabetes education in primary care, specifically; nutritional therapy counseling had the largest statistically significant impact on both weight loss and metabolic control. While, this meta-analysis focused on the diabetic population, the patients included in this analysis had both diabetes and hypertension. Control of both diseases can be assisted by weight reduction. 
There is evidence to support group weight loss programs. While, these programs may be costly to run, the benefits may outweigh the risks with regards to long-term complications. Rock et. al (2014) performed a randomized controlled trial with 227 overweight of obese with type-2 diabetes. The participants in the weight loss program, which included in person diet and exercise counseling, lost $7.8 \%$ of their initial weight as compared to $2.1 \%$ in the control group. The study found that a structured weight loss program resulted in greater weight loss and improved glycemic control in type-2 diabetes, improving cardiovascular risk factors.

There is research that demonstrates the increased risk of high blood pressure in patients with obstructive sleep apnea. In a prospective cohort study with 5,382 patients referred to sleep study, 922 of the patients initiated continuous positive airway pressure (CPAP) therapy. It was found that the presence of OSA was associated with an increased risk of incident hypertension (Marin, et. al, 2012). Also, there is evidence to support that it is more difficult to lower blood pressure if patients are suffering from OSA. In a randomized placebo controlled cross over study, the effects of four weeks of CPAP on 68 patients with hypertension were studied. The patients were not receiving hypotensive medications. Blood pressure was reduced by greater amounts in patients with intermittent nocturnal hypoxemia with the use of CPAP and systolic blood pressure was decreased by $4.0 \mathrm{~mm} \mathrm{Hg}$ and diastolic blood pressure by $5.0 \mathrm{~mm} \mathrm{Hg}$. For this reason, this clinic will begin screening all patients with co-morbid diagnosis for obstructive sleep apnea. Any patients with hypertension and possible sleep apnea will be referred for sleep study and CPAP, if indicated. 
Sleep apnea is more prevalent in patients with type-2 diabetes than patients without diabetes. Einhorn et al. (2007) completed a study with 279 adults with type- 2 diabetes. They found that there was a high prevalence of OSA in the diabetic patient population. They found that sleep apnea was associated with males, ages 62 and older, with a body mass index greater than or equal to $30 \mathrm{~kg} / \mathrm{m}^{2}$, snoring and reports of stopping breathing during sleep. Storgarrd et al. (2014) also found that approximately one third of patients with type-2 diabetes also had a co-morbidity of obstructive sleep apnea with a cross-sectional study including 200 participates. Therefore, it is even more important that patients with both hypertension and diabetes are screened for obstructive sleep apnea on a regular basis. Within the cohort of veterans in this study, patients with a diagnosis of hypertension without diabetes or chronic kidney disease, had a diagnosis of obstructive sleep apnea $41.7 \%$ of the time, compared to a prevalence of $50 \%$ of the patients with hypertensive patients with diabetes. This cohort of patients is reflective of the studies and research previously mentioned above.

\section{Implications for clinical practice}

Several future Doctor of Nursing Practice (DNP) students will implement the clinically significant outcomes and recommendations identified from this research. One student will begin screening patients with this co-morbidity for obstructive sleep apnea using either the STOP-Bang Screening tool or the Epworth Sleepiness Scale (ESS). This will help to identify patients that are suffering from OSA but have not been diagnosed and treated. Also, due to the large percentage of both overweight and obese patients in this data set, a student will implement the recommended interventions to reduce body 
mass index (BMI). The identified areas of concern, increased BMI and high prevalence of OSA will be addressed by these students and hopefully will then decrease blood pressure in these patients. Clinically, there is still a need for further intervention in this population, as there is work to do in order to meet blood pressure goal standards. With regards to future research, it would be interesting to look at the relationship between patients with both hypertension and diabetes and other psychosocial contributing factors such as anxiety, depression and post-traumatic stress disorder (PTSD). Also, it would be helpful to have a better understanding of how often patients actually use their CPAP machines and any interventions that may increase compliance.

There are many areas within this patient population in which further education could be beneficial. This could be evaluated with a pre and posttest to gain a better idea of how much these patients understand the diseases and the consequences of poor blood pressure control over time. There is an opportunity formation of new statewide or national policy that encourages lifestyle changes among these patients through the use of incentives. Due to the complications associated with uncontrolled hypertension, especially in the setting of diabetes, there can be financial savings long term if blood pressure is controlled.

\section{Conclusions}

There is a need for continued aggressive treatment of hypertension to maintain blood pressure at or below the recommended 140/90mmHg per the JNC-8 Hypertension Guidelines for hypertensive and diabetic patients. All patients with a co-morbidity of hypertension and diabetes should be referred for nutritional counseling. Also, the Dietary 
Approach to Stop Hypertension (DASH) diet should be recommended to these patients. Weight management should be discussed with these patients. Finally, patients with hypertension and diabetes should be screened for obstructive sleep apnea. 


\section{References}

Brown, et al. (1989) Effects of educational interventions in diabetes care: a meta-analysis of findings. Nursing Research 37:223-230

Centers for Disease Control and Prevention (2016) Hypertension. Retrieved from http://www.cdc.gov/nchs/fastats/hypertension.htm

Chung, F., Abdullah, H., \& Liao, P. (2016). Stop-bang questionnaire: a practical approach to screen for obstructive sleep apnea. Chest,149(3) 631- 638.

Coughlin, S., Mawdsley, L., Mugarza, J., Wilding, J., \& Calverley, P. (2007) Cardiovascular and metabolic effects of CPAP in obese males with OSA. European Respiratory Journal. 29, 720-727. doi: 10.1183/09031936.00043306

Einhorn, D., Stewart, D., Erman, M., Gordon, N., Philis-Tsimikas, A., \& Casal, E., (2007). Prevalence of sleep apnea in a population of adults with type 2 diabetes mellitus. American Association of Clinical Endocrinologists. 13(4) 355-362

Faccenda, J., Mackay, T., Boon, N., \& Douglas, N. (2001) Randomized placebocontrolled trial of continuous positive airway pressure on blood pressure in the sleep apnea-hypopnea syndrome. American Journal of Respiratory and Critical Care Medicine .163, (2), 344-348.

Goodson, B., Wung, S., \& Archbold, K. (2012). Obstructive sleep apnea hypopnea syndrome and metabolic syndrome: A synergistic cardiovascular risk factor. Journal of the American Academy of Nurse Practitioners 24 695-703.

James PA, Oparil S, Carter BL, Cushman WC, Dennison-Himmelfarb C, Handler J, Lackland DT, LeFevre ML, MacKenzie TD, Ogedegbe O, Smith SC, Svetkey LP, Taler SJ, Townsend RR, Wright JT, Narva AS, Ortiz E. 2014 Evidence-Based 
Guideline for the Management of High Blood Pressure in Adults- Report From the Panel Members Appointed to the Eighth Joint National Committee (JNC 8). JAMA. 2014;311(5):507-520. doi:10.1001/jama.2013.284427

Malauti, M. (2016). Evidence for the effectiveness of clinical nutrition therapy in diabetes mellitus type 2: management in primary care. Journal of the Australian Tradition-Medicine Society 22(2) 74- 82

Marin JM, Agusti A, Villar I, Forner M, Nieto D, Carrizo SJ, Barbé F, Vicente E, Wei Y, Nieto FJ, \& Jelic S. (2013) Association Between Treated and Untreated Obstructive Sleep Apnea and Risk of Hypertension. JAMA. 307 (20) 2169-2176. doi:10.1001/jama.2012.3418

Mbata, G., \& Chukwuka, J. (2013) Obstructive sleep apnea hypopnea syndrome. Annals of Medical and Health Science Research. 2(1): 74-77 doi: 10.4103/2141$\underline{9248.96943}$

McClinchy, J., Dickinson, A., Barron, D., \& Thomas, H. (2011). Practitioner and lay perspectives of the services provision of nutrition information leaflets in primary care. Journal of Human Nutrition and Dietetics. 24. 552-559.

National Heart, Lung, and Blood Institute. (2015) U.S Department of Health \& Human Services. Health benefits of the DASH eating plan. Retrieved from https://www.nhlbi.nih.gov/health/health-topics/topics/dash/benefits

Rock, C., Flatt, S., Pakiz, B., Taylor, K., \& Leone,A., (2014). Weight loss, glycemic control, and cardiovascular disease risk factors in response to differential diet composition in a weight loss program in type 2 diabetes: a randomized controlled trial. Diabetes Care 37(6): 1573-1580 
Roux, L., Ubach, C., Donaldson, C. \& Ryan, M. (2004). Valuing the benefits of weight loss programs: an application of the discrete choice experiment. Obesity Research 12 (8) 1342- 1351.

Storgaard, H., Mortensen, B., Almdal, T., Laub, M., \& Tarnow, L. (2014) At least one in three people with type 2 diabetes mellitus referred to a diabetes centre has symptomatic obstructive sleep apnoea. Diabetic Medicine UK, 1460-1467.

Vana, K., Silva, G., \& Goldberg, R. (2013). Predictive abilities of the stop-band and Epworth sleepiness scale in identifying sleep clinic patients at high risk for obstructive sleep apnea. Research in Nursing \& Health. 36; 84-94. 
Table 1. Twenty-One Non-Black Patients w/ Out of Control Blood Pressure

\begin{tabular}{|c|c|}
\hline Number of Patients & Number of Anti-Hypertensives \\
\hline 2 patients & Single therapy \\
8 patients & Double therapy \\
4 patients & Triple therapy \\
5 patients & Quadruple therapy \\
2 patient & \\
\hline
\end{tabular}

Figure 1. Body Mass Index of 102 Hypertensive \& Diabetic Patients

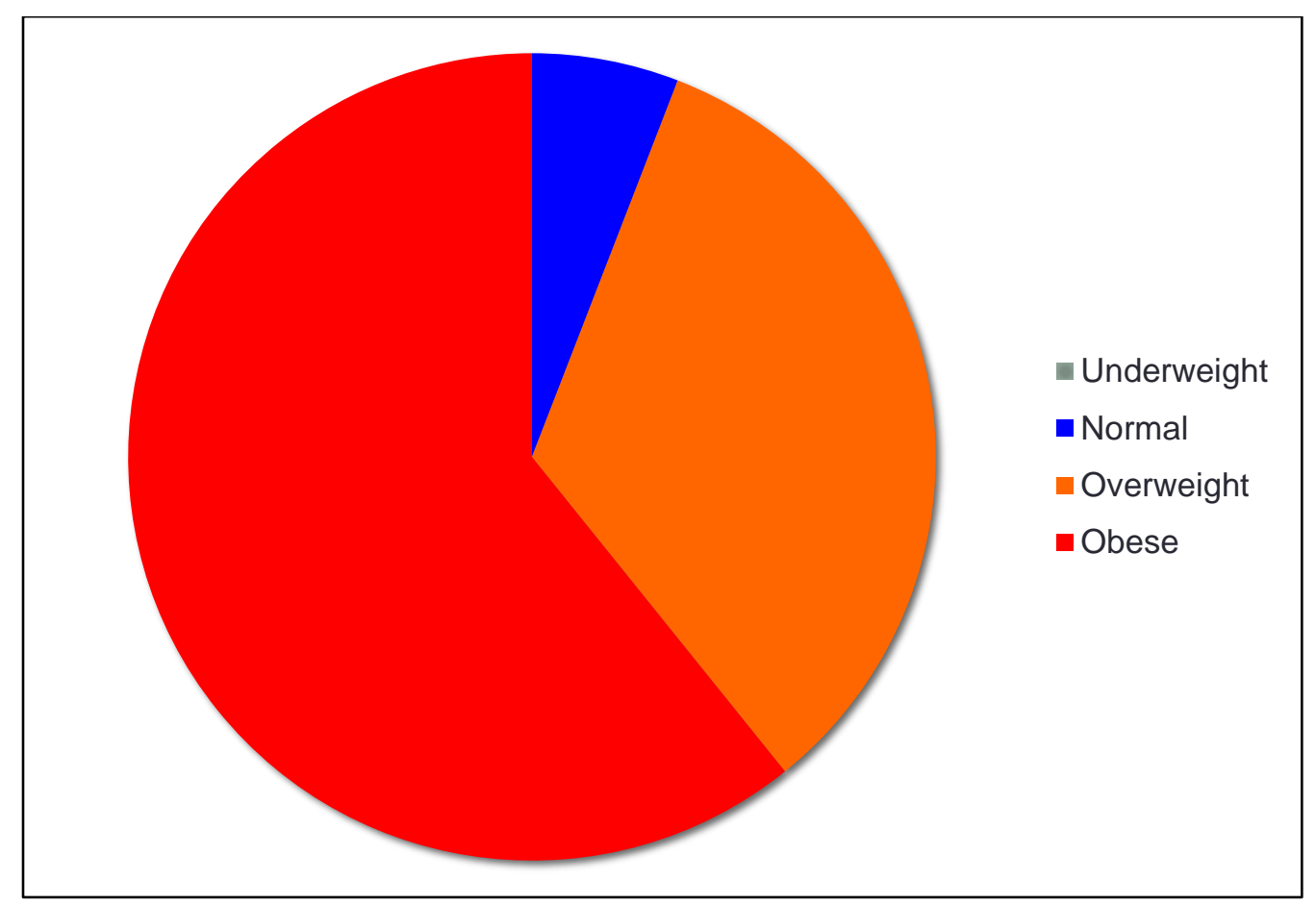


Figure 2. Hypertensive and Diabetic Patients with or without Obstructive Sleep Apnea

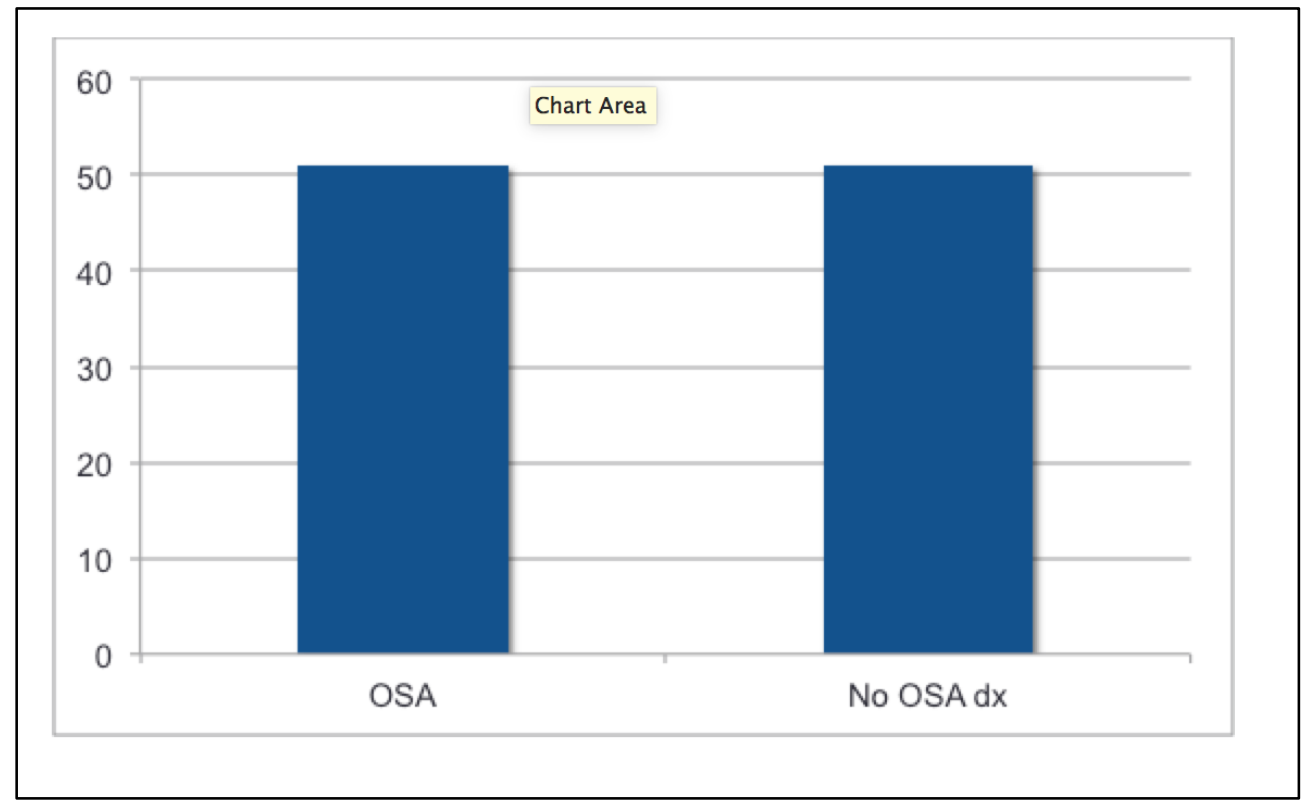

Figure 3. Picture Aid of Clinical Significance

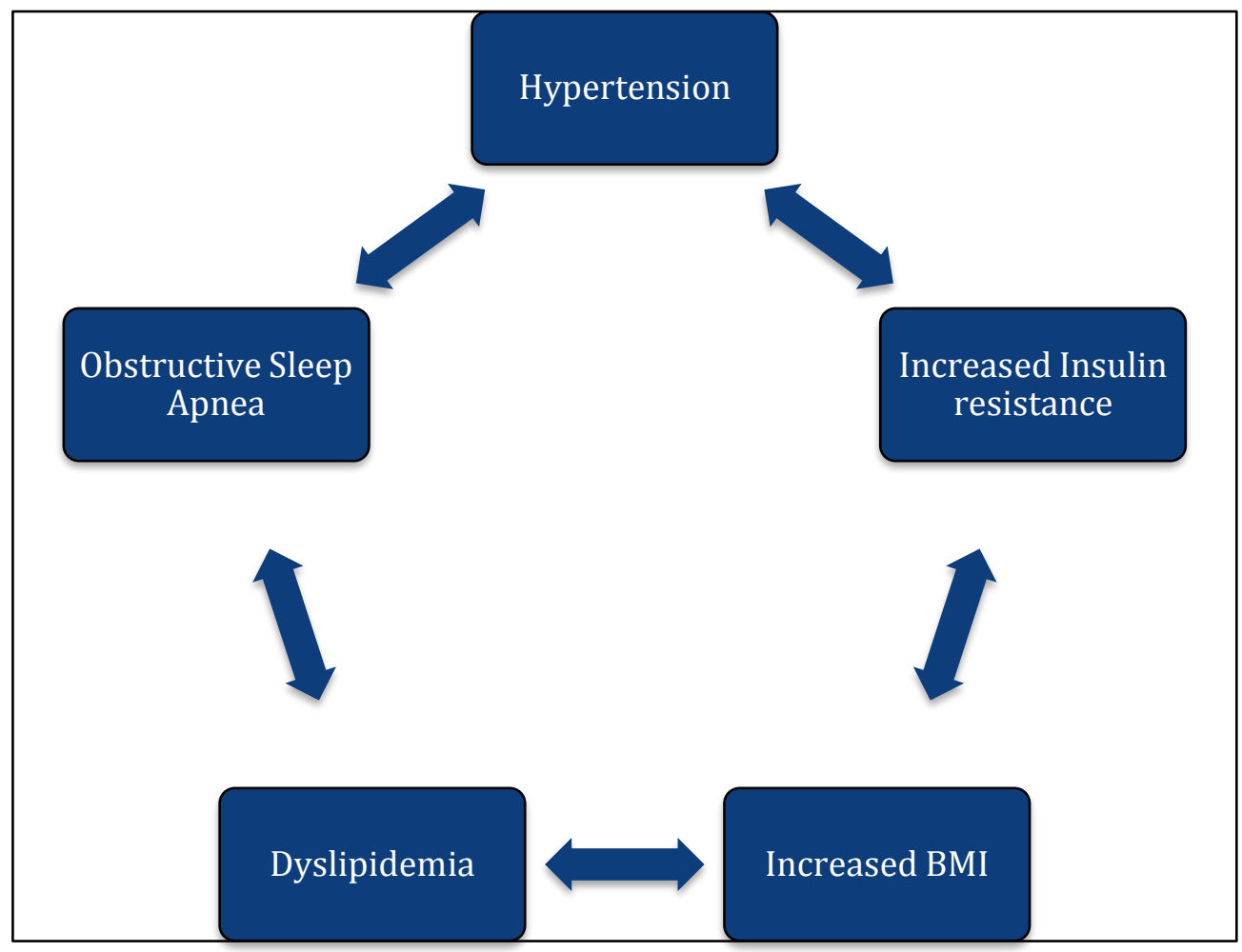

